\title{
De KAOS para SysML na Modelagem de Sistemas Embarcados: Uma Revisão da Literatura
}

\author{
Timóteo Gomes ${ }^{1}$, Fernanda Alencar ${ }^{1}$ e Aêda Sousa ${ }^{1}$ \\ ${ }^{1}$ Universidade Federal de Pernambuco, Departamento de Eletrônica e Sistemas, 50.740-560, \\ Recife - PE- Brasil \\ timoteogomes@yahoo.com.br, fernandaalenc@gmail.com, \\ aedasousalgmail.com
}

\begin{abstract}
Na modelagem de sistemas, de modo em geral, não se leva em consideração as razões e intenções que determinam as funcionalidades desses sistemas. A Engenharia de Requisitos Orientada a Objetivos (do inglês, Goal Oriented Requirements Engineering - GORE) visa resolver esse problema. Nesse contexto, está KAOS, que chega aos requisitos, pretendendo dar respostas às questões "Por quê?", "Quem?" e "Quando?" sobre os objetivos/metas previamente declarados. Por outro lado, no desenvolvimento de sistemas complexos, nos quais os sistemas embarcados estão inseridos, utiliza-se a linguagem de modelagem SysML, mas que como UML, não dá muita atenção a dimensão das razões e intenções. Desta feita, pretende-se integrar KAOS a SysML através do mapeamento de seus quatro modelos para os diagramas da SysML. Alguns desses modelos são naturais à UML, no entanto SysML tem outras dimensões que precisam ser consideradas, sobretudo os novos diagramas: o de requisitos e o paramétrico. Como forma de verificar o grau de correção do pretendido mapeamento, pensa-se utilizar simulações através da modelagem em redes de Petri. Neste artigo são apresentados os primeiros resultados da revisão sistemática da literatura para a identificação de abordagens similares e de problemas ainda em aberto no contexto da modelagem de sistemas embarcados.
\end{abstract}

Keywords: SysML. KAOS. Rede de Petri. Sistemas Embarcados.

\section{Introdução}

Sistemas embarcados estão remodelando a forma como as pessoas vivem, trabalham e se divertem [1]. Esses sistemas, usados em transportes terrestres, medicina, eletrônica de consumo, ambientes aviônicos, robótica dentre outros, geralmente recebem dados de entrada a partir de sensores, processam esses dados usando microcontroladores ou microprocessadores, e enviam os resultados para atuadores e/ou interface com os usuários [2]. A complexidade inerente aos diferentes domínios, aliada à necessidade de encontrar defeitos ou faltas de forma rápida e precisa, bem como as razões e intenções são alguns dos fatores importantes a serem considerados no desenvolvimento desses sistemas [3]. Nesse contexto, às especificidades dos diversos tipos de sistemas, 
levam a uma complexidade na compreensão dos requisitos, que devem ser elicitados e traduzidos em funcionalidades. A fase inicial, de definição dos requisitos, em todo projeto é considerada a fase estrategicamente mais sensível. Isso por causa da sua subjetividade, com alta probabilidade de erro e/ou ausência de requisito, fazendo com que uma formalização que abrange as etapas de obtenção, especificação e análise de requisitos seja inviável nesta fase [4]. Neste sentido, existem pesquisas [4, 5] que antecipam essa formalização para as fases iniciais, através do uso de linguagens diagramáticas como UML e SysML.

Ao se tratar da engenharia de requisitos em sistemas embarcados, verificou-se que o perfil SysML foi especialmente concebido pelo Object Management Group (OMG) para a análise e especificação de sistemas complexos. Com esse perfil, é permitida a captura de requisitos e a manutenção de links de rastreabilidade entre esses e diagramas de projeto resultantes da fase de projeto do sistema [6].

Por outro lado, além da formalidade trazida pelo uso de perfis, faz-se necessário considerar as razões e intenções capazes de determinar alternativas e configurações de funcionalidades nos sistemas pretendidos, bem como as responsabilidades. Nesse contexto, tem-se a popular abordagem de Engenharia de Requisitos Orientada a Objetivos (do inglês, Goal Oriented Requirements Engineering - GORE) [7]. Em particular, o framework KAOS tem sido foco de alguns trabalhos [8, 9] nos últimos anos. Com KAOS pretende-se a construção de um modelo de requisitos que descreve o problema a ser resolvido e as restrições que devem ser superadas quando a solução é implementada [10]. Além de fornecer uma linguagem para elicitar requisitos, KAOS pretende dar respostas a questões como "Por quê?", "Quem?" e "Quando?” sobre os objetivos/metas previamente declarados [11]. KAOS envolve uma série de redes semânticas para a modelagem conceitual dos objetivos, hipóteses, agentes, objetos e operações do sistema [8]. A análise se dá por meio de técnicas de refinamento de objetivos/metas, detecção de resolução de conflitos e delegação de responsabilidades a agentes [12]. Para tal, o KAOS propõe quatro modelos: de objetivos; de responsabilidade; de objetos; e, de operações.

Neste trabalho, busca-se apresentar o andamento da pesquisa com relação à integração de KAOS com o perfil SysML. A partir da inserção de metas, os novos diagramas do SysML, de requisitos e de parâmetros, deverão ser verificados. Para tanto, será utilizada a abordagem de Salmon [4], que propôs um método formal de modelagem, análise e verificação de requisitos, partindo de uma representação semi-formal, usando UML, e, posteriormente, o formalismo das redes de Petri para proceder a análise e verificação. A rede de Petri pode ser vista como um formalismo visual que pode explicar o comportamento físico de um sistema e seus componentes [5]. Além do seu poder de representação, as redes de Petri também permitem a análise do sistema modelado, identificando conflitos, ciclos, comportamentos inesperados e deadlocks [13].

O trabalho encontra-se estruturado da seguinte forma: na Seção 2 são apresentados os objetivos da pesquisa; na Seção 3, apresenta-se o andamento do trabalho e as contribuições esperadas; por fim, na Seção 4 são apresentadas as conclusões e trabalhos futuros. 


\section{Objetivos da pesquisa}

O objetivo principal desta pesquisa é realizar a integração de GORE, através de KAOS, à SysML, através da proposição de heurísticas de mapeamento automático de seus elementos. Assim, será possível uma descrição textual (SysML) que contemple razões/intenções e responsabilidades (KAOS) na modelagem de requisitos no contexto de Sistemas Embarcados. Ao mesmo tempo, o mapeamento será integrado ao ambiente de redes de Petri, como forma de verificação do mesmo. Na Figura 1 são descritas as etapas programadas para o desenvolvimento da pesquisa.

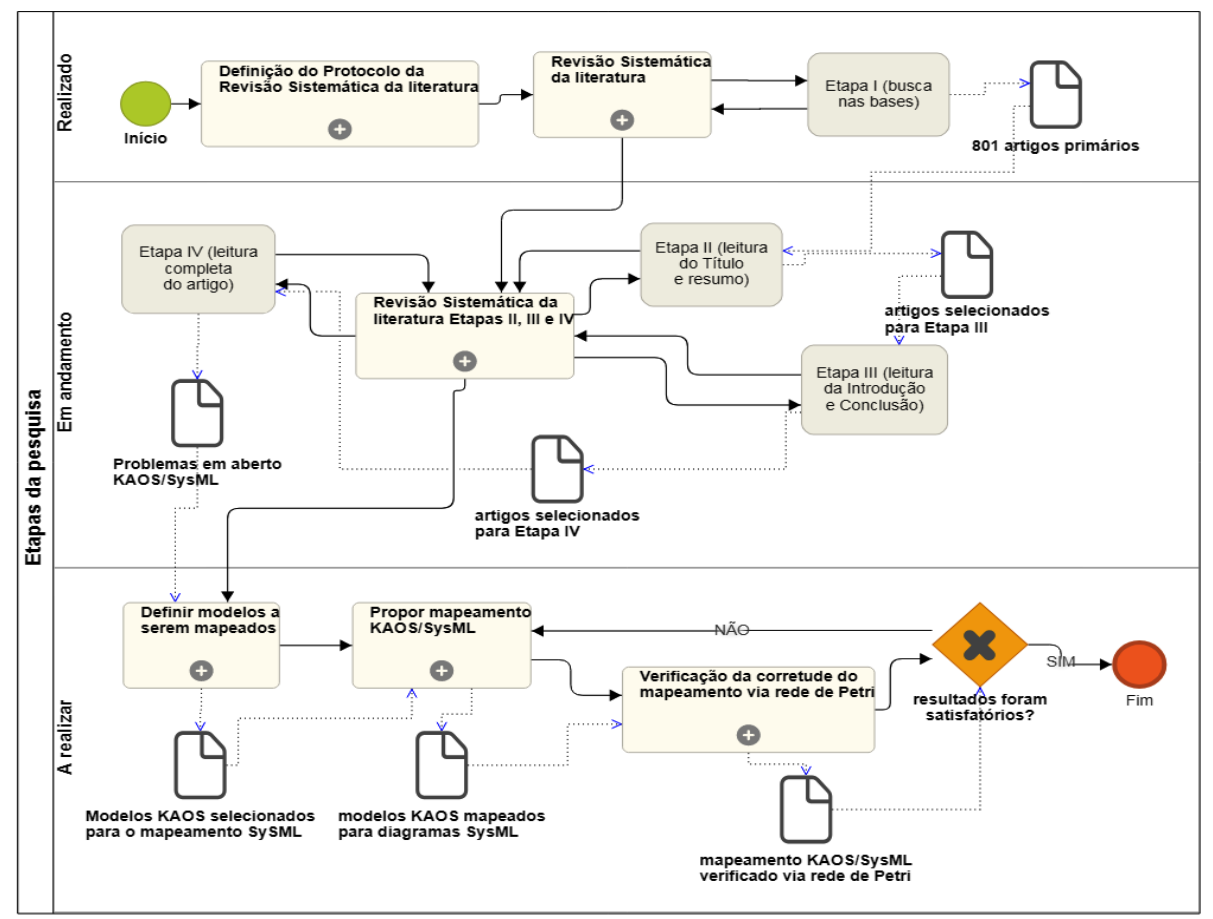

Fig. 1. Etapas da pesquisa (Autor, 2018)

Conforme a Figura 1, já foram realizadas a definição do Protocolo da Revisão Sistemática da Literatura (RSL), documento norteador de toda a RSL (questões de pesquisa, bases de busca e etc), e a Etapa 1 da RSL, etapa responsável pela realização das pesquisas nas bases de acordo com as strings de busca definidas. O estágio em andamento demonstra que a Etapa II (leitura do título e resumo) da RSL está em curso, dando seguimento as Etapas III e IV, permitindo levantar os problemas em aberto no universo KAOS/SysML. Após conclusão dessa fase, serão realizados ainda os processos de definição dos modelos/elementos de KAOS que serão mapeados para SysML, isso baseado nos resultados obtidos na RSL. Em seguida será realizado mapeamento propriamente dito de KAOS/SysML (técnicas de mapeamentos, ferramentas e etc). 
Uma vez concluído o mapeamento, será realizado o processo de verificação do nível de corretude do referido mapeamento, utilizando para isso redes de Petri.

\section{Andamento do trabalho e as contribuições}

Nesta seção são apresentados o andamento do trabalho e a metodologia adotada para este trabalho assim como as etapas já realizadas até o momento. Também são descritas as possíveis contribuições advindas desta pesquisa.

\subsection{Revisão Sistemática da Literatura}

Com o objetivo de identificar os problemas em aberto no que diz respeito ao uso de KAOS associado à SysML e Redes de Petri no desenvolvimento de Sistemas Embarcados, foi elaborado um protocolo de RSL, seguindo-se diretrizes consagradas na literatura [14]. Nesse protocolo, procura-se responder a sete questões de pesquisa, sendo apresentadas três delas na Tabela 1. Foram definidas as palavras chaves (e seus sinônimos) e alinhadas em três strings de busca, dentre elas, a string 03, descrita na Tabela 2, não apresentando as demais por limitação de espaço.

Tabela 1. Questões de Pesquisa

\begin{tabular}{cc}
\hline \# & Questão de Pesquisa \\
\hline QP1 & Existe algum mapeamento sintático e/ou semântico de KAOS para SysML? \\
QP2 & Existe mapeamento dos elementos de SysML para Rede de Petri? \\
QP3 & Quais os desafios/problemas identificados na literatura relacionadas à SysML \\
em relação a verificação/validação em sistemas embarcados?
\end{tabular}

Fonte: (Autor, 2018)

Realizou-se buscas em 8 bases de dados, conforme Figura 2. Na Etapa 1 foram identificados 801 artigos primários cujos resultados podem ser vistos na Figura 2. Atualmente o trabalho encontra-se na Etapa II (leitura da introdução e resumo), em andamento. Após concluir a Etapa II, será dado prosseguimento a revisão, realizando-se as Etapas III (leitura da introdução e conclusão de cada estudo selecionado na etapa II) e IV (os trabalhos oriundos da etapa III serão lidos por completo e aplicado os critérios de qualidade). 


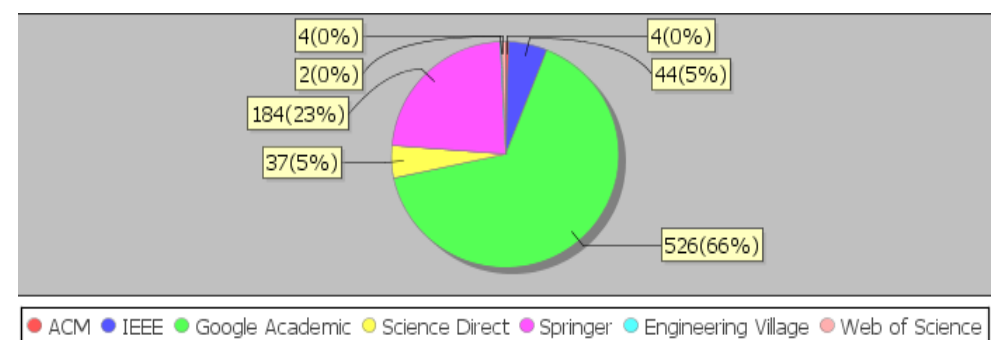

Fig. 2. Artigos primários identificados na Etapa I (Autor, 2018)

\subsection{Contribuições esperadas}

A principal contribuição do trabalho serão as heurísticas de mapeamento automático dos elementos de KAOS para SysML, com isso enriquece-se a descrição textual (SysML) e com as razões, objetivos e responsabilidades (KAOS) dos requisitos na modelagem de Sistemas Embarcados. Pretende-se resolver a problemática da semântica entre as relações da SysML usando KAOS [15]. Como em KAOS os NFRs são considerados apenas no nível arquitetural, SysML permitirá a avaliação de opções alternativas, risco e análise de conflitos com antecedência [15]. Com SysML é possível verificar relações de rastreabilidade, o que não é tão explícita em KAOS [15;16]. A verificação de corretude do mapeamento dos modelos de KAOS/SysML via rede de Petri poderá estender mapeamentos SysML/rede de Petri [16,17], assim como propor novos mapeamentos SysML/rede de Petri.

\section{Conclusões e trabalhos futuros}

Até a fase atual da revisão sistemática, não foram encontrados trabalhos que mencionem o mapeamento de elementos de KAOS para diagramas da SysML. Foram identificados até o momento, alguns trabalhos relacionados [4, 8, 17, 18].

Como trabalhos futuros serão realizadas as etapas de: conclusão da RSL, definiç̧ão dos modelos de KAOS e diagramas da SysML que serão mapeados e a verificação do mapeamento via rede de Petri. A análise via rede de Petri, será baseada em trabalhos como $[4,17,18]$, onde serão reaplicados estes estudos de casos já validados de migração de diagramas de SysML/UML para rede de Petri, possibilitando a verificação da exatidão dos resultados advindo da modelagem com a proposta deste trabalho.

\section{$5 \quad$ Agradecimentos}

Agradecemos a UFPE e UPE pela disponibilização dos recursos necessários à elaboração do trabalho, assim como por todo apoio prestado. 


\section{Referências}

1. Ribeiro, Q. A. D. D. S.: Uma técnica baseada em SysML para modelar a arquitetura de sistemas embarcados de tempo real (2017).

2. M. Broy, "Requirements Engineering for Embedded Systems," Work. Form. Des. Saf. Crit. Embed. Syst. FEmSys 1997, pp. 1-11 (1997).

3. Pereira, T., Albuquerque, D., Sousa, A., Alencar, F. and Castro, J.: "Retrospective and trends in requirements engineering for embedded systems: A systematic literature review," in Proceedings of the XX Iberoamerican Conference on Software Engineering (2017).

4. Salmon, A. Z. O.: Modelagem e análise de requisitos de sistemas automatizados usando UML e Redes de Petri. Tese de Doutorado. Universidade de São Paulo (2017).

5. Friedenthal, S., Moore, A., Steiner, R.: A practical guide to SysML: the systems modeling language. Morgan Kaufmann (2014).

6. Friedenthal, S., Moore, A., Steiner, R.: A practical Guide to SysML. Morgan Kaufmann OMG Press (2008).

7. Parveen, S.; Imam, A.: Analysis of different techniques of gore (goal oriented requirement engineering). Global Sci-Tech, Al-falah School of Engineering and Technology, v. 9, n. 1, p. 22-36 (2017)

8. Martinez Silva, J.: Modelagem e análise de conhecimento para planejamento automático: uma abordagem baseada em GORE e redes de Petri (Doutorado, USP) (2017).

9. Engelsman, W., \& Wieringa, R., Goal-oriented requirements engineering and enterprise architecture: Two case studies and some lessons learned. In International Working Conference on Requirements Engineering: Foundation for Software Quality (pp. 306-320). Springer, Berlin, Heidelberg (2012).

10. Watanabe, N.: Uma Proposta de Modelagem Orientada a Personas para o Modelo de Objetivo Orientado a Contexto / Naiara Watanabe. Brasília: UnB (2016).

11. Lamsweerde, A. V.: Goal-oriented requirements engineering: From system objectives to uml models to precise software specifications. Proceedings of the 25th International Conference on Software Engineering, IEEE Computer Society, p. 744-745 (2003).

12. Santos, C. O. Dos.: Aplicação do modelo $i^{*}$ em um processo de análise de requisitos orientado a metas. Faculdade de Ciências Exatas e da Natureza - UNIMEP (2006).

13. Silva, C. E. A. D.: Desenvolvimento de Biblioteca para Aplicações de PNRD e PNRD Invertida Embarcadas em Arduino (2017).

14. B. Kitchenham, O. P. Brereton, D. Budgen, M. Turner, J. Bailey, and S. Linkman, "Systematic literature reviews in software engineering - A systematic literature review," Inf. Softw. Technol., vol. 51, no. 1, pp. 7-15, 2009

15. Ahmad, M., Belloir, N., \& Bruel, J. M.: Modeling and verification of functional and nonfunctional requirements of ambient self-adaptive systems. Journal of Systems and Software, 107, 50-70 (2015).

16. Tueno, S., Laleau, R., Mammar, A., \& Frappier, M.: The SysML/KAOS Domain Modeling Approach. arXiv preprint arXiv:1710.00903 (2017).

17. Carneiro, E., Maciel, P., Callou, G., Tavares, E., \& Nogueira, B.: Mapping sysml state machine diagram to time petri net for analysis and verification of embedded real-time systems with energy constraints. In Advances in Electronics and Micro-electronics. ENICS'08. International Conference on (pp. 1-6). IEEE (2008).

18. Andrade, E., Maciel, P., Callou, G., \& Nogueira, B.: A methodology for mapping sysml activity diagram to time petri net for requirement validation of embedded real-time systems with energy constraints. In Digital Society. ICDS'09. Third International Conference on (pp. 266-271). IEEE (2009). 